\title{
Development of a Simulation Model for the Vacuum Pressure Swing Adsorption Process to Sequester Carbon Dioxide from Coalbed Methane
}

\author{
Daeho Ko*
}

Global Engineering Division of GS Engineering \& Construction, Gran Seoul, 33, Jong-ro, Jongno-gu, Seoul 110-130, Korea

\footnotetext{
* To whom correspondence should be addressed. Tel: +82 22154 6171, Email: daeho.ko@gsconst.co.kr or daehoko@hotmail.com
} 


\section{Supporting Figure Captions}

Figure S1.1. Temperature Profiles of Experiments and Simulations at Cyclic Steady State (CSS) (Case 1b)

(a) Case $1 b(z=0.3)$

(b) Case $1 b(z=0.7)$

Figure S1.2. Temperature Profiles of Experiments and Simulations at Cyclic Steady State (CSS) (Case 2b)

(a) Case $2 \mathrm{~b}(\mathrm{z}=0.3)$

(b) Case $2 b(z=0.7)$

Figure S1.3. Temperature Profiles of Experiments and Simulations at Cyclic Steady State (CSS) (Case 3b)

(a) Case $3 b(z=0.3)$

(b) Case $3 b(z=0.7)$

Figure S2. Effect of Packing Bed Length Change

Figure S3. Effect of Inner Diameter Change

Figure S4. Effect of Adsorption Pressure Change

Figure S5. Effect of Vacuum Purge Pressure Change

Figure S6. Effect of Temperature Change

Figure S7. Effect of Operating Step Time Change

Figure S8. Effect of Feed Flow Rate 


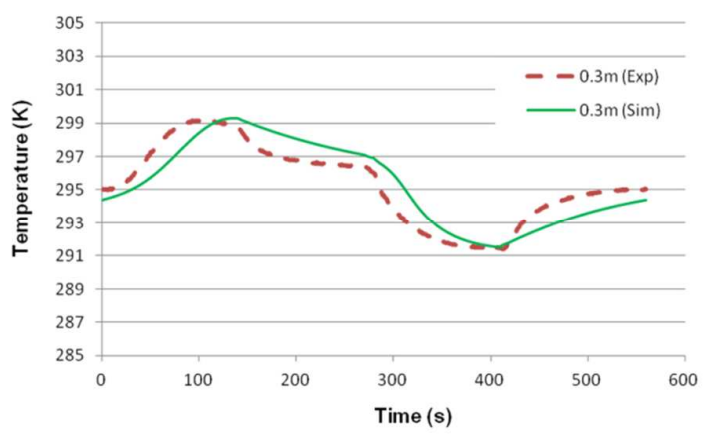

(a) Case $1 \mathrm{~b}(\mathrm{z}=0.3)$

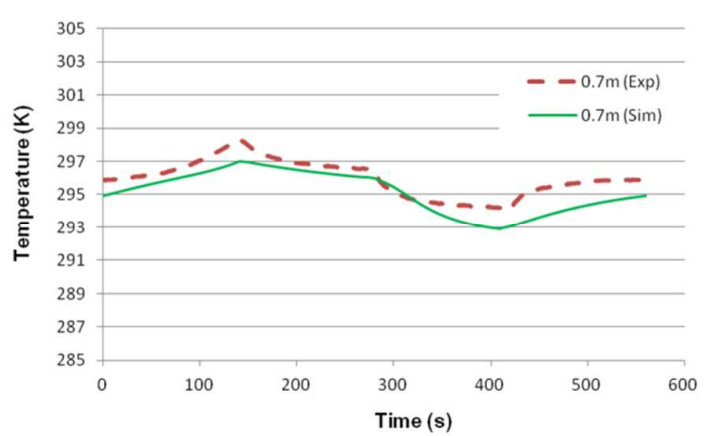

(b) Case $1 b(z=0.7)$

Figure S1.1. Temperature Profiles of Experiments and Simulations at Cyclic Steady State (CSS) (Case 1b) 


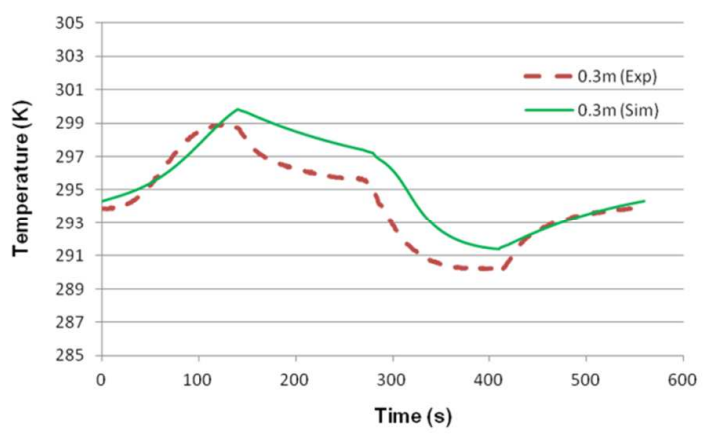

(a) Case $2 b(z=0.3)$

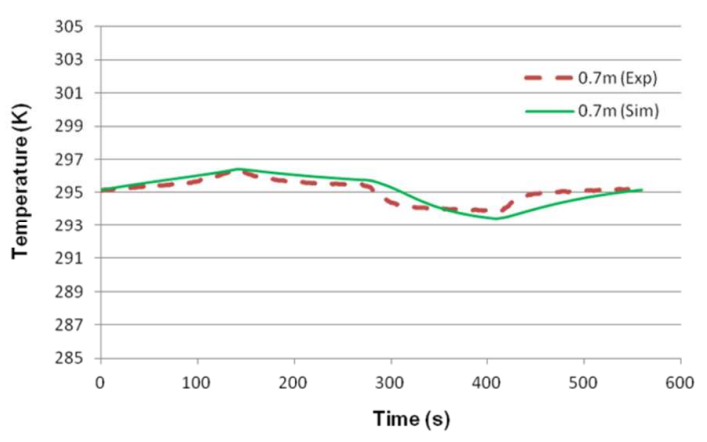

(b) Case $2 b(z=0.7)$

Figure S1.2. Temperature Profiles of Experiments and Simulations at Cyclic Steady State (CSS) (Case 2b) 


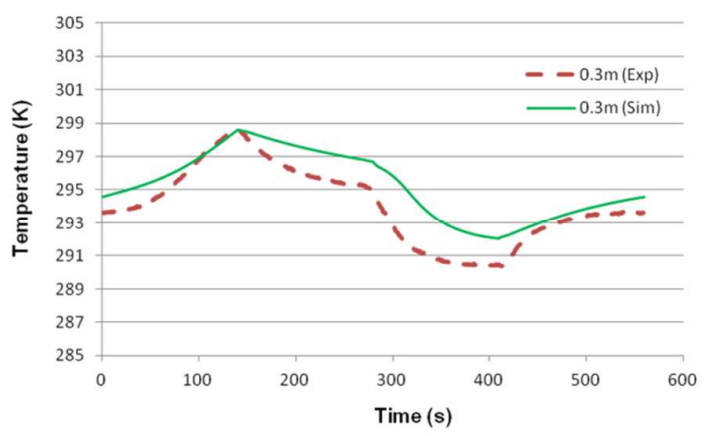

(a) Case $3 b(z=0.3)$

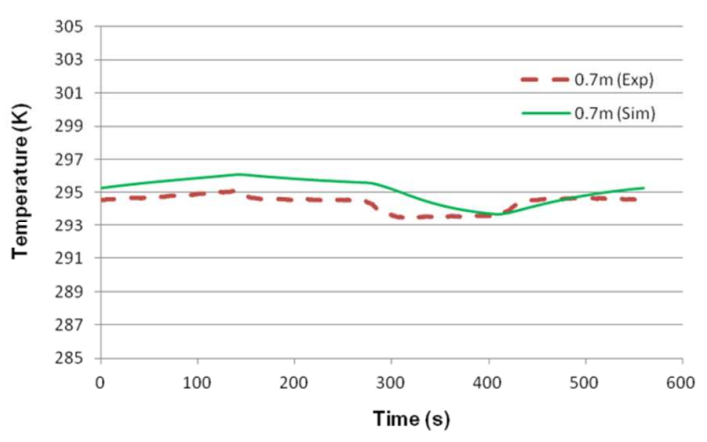

(b) Case $3 b(z=0.7)$

Figure S1.3. Temperature Profiles of Experiments and Simulations at Cyclic Steady State (CSS) (Case 3b) 


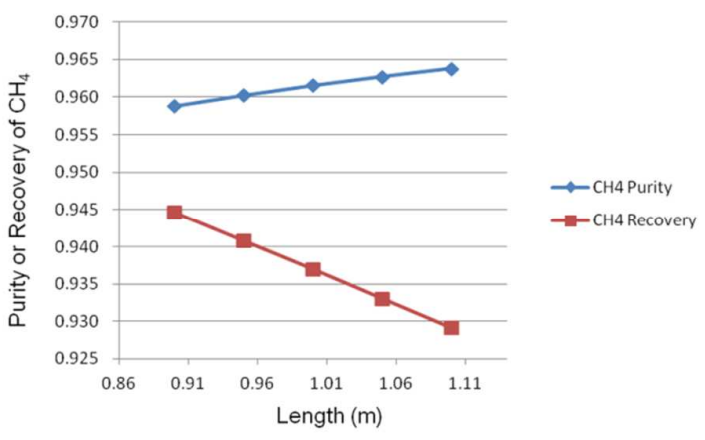

Figure S2. Effect of Packing Bed Length Change

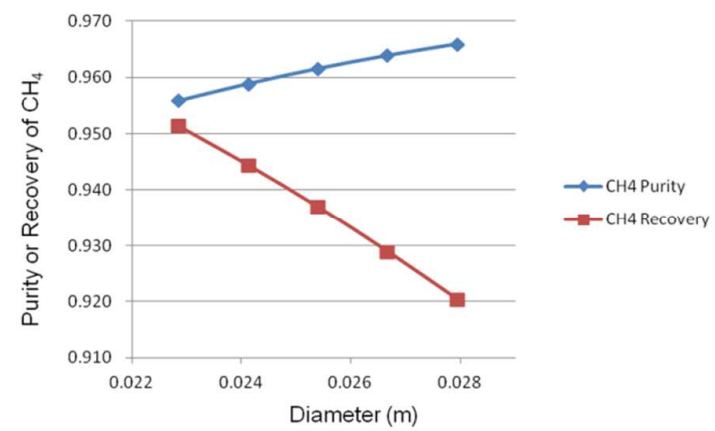

Figure S3. Effect of Inner Diameter Change

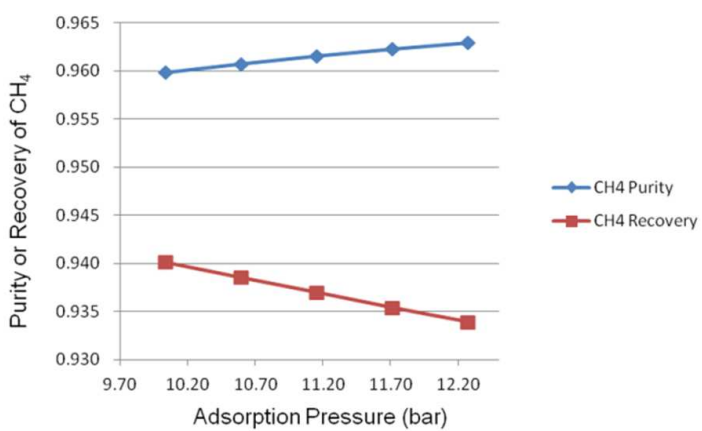

Figure S4. Effect of Adsorption Pressure Change

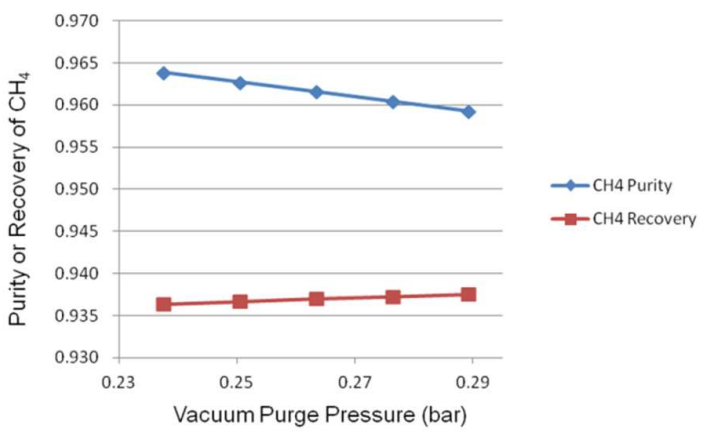

Figure S5. Effect of Vacuum Purge Pressure Change 


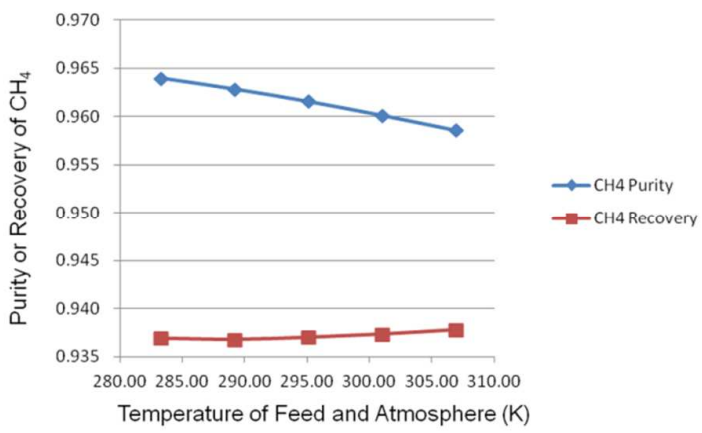

Figure S6. Effect of Temperature Change

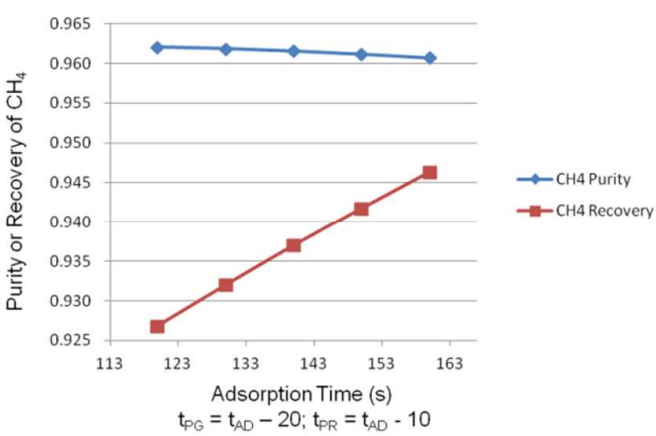

Figure S7. Effect of Operating Step Time Change

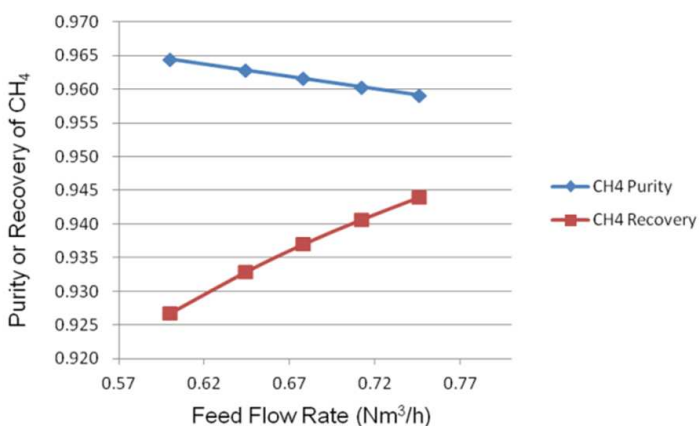

Figure S8. Effect of Feed Flow Rate 


\section{List of Supporting Tables}

Table S1. Specifications for feed to LNG plants and pipeline gas

Table S2. Parameters of adsorbent, Shirasagi MSC 3K-172

Table S3. Four bed twelve operating step sequence and times

Table S4. Isotherm parameters and adsorption heat

Table S5. Mass transfer coefficients of each component in the LDF model

Table S6.1. Boundary conditions (molar fraction)

Table S6.2. Boundary conditions (pressure)

Table S6.3. Boundary conditions (molar flow rate)

Table S6.4. Boundary conditions (temperature)

Table S6.5. Boundary conditions (interstitial gas velocity)

Table S7. Simulation results of conventional approach (II) as to the various values of " $\alpha$ " 
Table S1. Specifications for feed to LNG plants and pipeline gas

\begin{tabular}{|c|c|c|}
\hline Impurity & Feed to LNG plants & Pipeline Gas \\
\hline $\mathrm{H}_{2} \mathrm{O}$ & $<0.1 \mathrm{ppmv}$ & $150 \mathrm{ppmv}$ \\
\hline $\mathrm{H}_{2} \mathrm{~S}$ & $<4 \mathrm{ppmv}$ & $5.7-22.9 \mathrm{mg} \mathrm{Sm}^{-3}$ \\
\hline $\mathrm{CO}_{2}$ & $<50 \mathrm{ppmv}$ & $3-4 \mathrm{vol.} \%$ \\
\hline Total Sulfur & $<20 \mathrm{ppmv}$ & $115-419 \mathrm{mg} \mathrm{Sm}^{-3}$ \\
\hline $\mathrm{N}_{2}$ & $<1 \mathrm{vol.} \%$ & - \\
\hline $\mathrm{Hg}$ & $<0.01 \mathrm{mg} / \mathrm{Nm}^{3}$ & - \\
\hline $\mathrm{C}_{4}$ & $<2 \mathrm{vol.} \%$ & - \\
\hline $\mathrm{C}_{5+}$ & $<0.1 \mathrm{vol} \% \%$ & - \\
\hline Aromatics & $<2 \mathrm{ppmv}$ & - \\
\hline Total Sulfur $=\mathrm{H}_{2} \mathrm{~S}+$ carbonyl sulphide, COS + organic sulphur \\
\hline
\end{tabular}

Table S2. Parameters of adsorbent, Shirasagi MSC 3K-172

\begin{tabular}{|l|l|}
\hline Parameters & Values \\
\hline Bed porosity & $0.35 \sim 0.38$ \\
\hline Particle porosity & $0.46 \sim 0.5$ \\
\hline Heat capacity & $880 \mathrm{Jkg}^{-1} \mathrm{~K}^{-1}$ \\
\hline Particle density & $680 \sim 700 \mathrm{~kg} \mathrm{~m}^{-3}$ \\
\hline Particle diameter & $1.8 \mathrm{~mm}$ \\
\hline Filling Bulk Density & $680 \sim 730 \mathrm{kgm}^{-3}$ \\
\hline
\end{tabular}

Table S3. Four bed twelve operating step sequence and times

\begin{tabular}{|c|c|c|c|c|c|c|c|c|c|c|c|c|}
\hline STEP NO. & 1 & 2 & 3 & 4 & 5 & 6 & 7 & 8 & 9 & 10 & 11 & 12 \\
\hline TIME(sec) & 10 & 120 & 10 & 10 & 120 & 10 & 10 & 120 & 10 & 10 & 120 & 10 \\
\hline Bed 1 & AD & AD & AD & EQ1 & RE & EQ2 & BD & PG & EQ2 & EQ1 & PR & PR \\
\hline Bed 2 & BD & PG & EQ2 & EQ1 & PR & PR & AD & AD & AD & EQ1 & RE & EQ2 \\
\hline Bed 3 & EQ1 & PR & PR & AD & AD & AD & EQ1 & RE & EQ2 & BD & PG & EQ2 \\
\hline Bed 4 & EQ1 & RE & EQ2 & BD & PG & EQ2 & EQ1 & PR & PR & AD & AD & AD \\
\hline
\end{tabular}

$A D$ : adsorption, $E Q$ : pressure equalization, $R E$ : repose, $B D$ : blow down, $P G$ : purge, $P R$ : pressurization 
Table S4. Isotherm parameters and adsorption heat

\begin{tabular}{|c|c|c|c|c|c|}
\hline & $\begin{array}{c}\mathbf{q}_{\mathbf{s a}} \\
\left(\mathbf{m o l e ~ g}^{-\mathbf{1}}\right)\end{array}$ & $\begin{array}{c}\mathbf{q}_{\mathbf{s b}} \\
\left(\mathbf{m o l e ~} \mathbf{~ k g}^{-\mathbf{1}}\right)\end{array}$ & $\begin{array}{c}\mathbf{b}_{\mathbf{0}} \\
\left(\mathbf{b a r}^{-\mathbf{1}}\right)\end{array}$ & $\begin{array}{c}\mathbf{D}_{\mathbf{E}} \\
(\mathbf{K})\end{array}$ & $\begin{array}{c}\triangle \mathbf{H} \\
\left(\mathbf{J} \mathbf{~ m o l e}^{-\mathbf{1}}\right)\end{array}$ \\
\hline $\mathrm{CH}_{4}$ & -0.0018 & 1.502 & 0.413302326 & -238 & 1637.227 \\
\hline $\mathrm{CO}_{2}$ & 0.0004 & 0.9374 & 0.000755814 & 2250.1 & 8763.67 \\
\hline $\mathrm{N}_{2}$ & 0.0019 & -0.00376 & 0.000335559 & 1937.8 & 6463.01 \\
\hline
\end{tabular}

Table S5. Mass transfer coefficients of each component in the LDF model

\begin{tabular}{|c|c|}
\hline Mass transfer coefficient & Value \\
\hline$k_{C H 4}$ & $5.35 \times 10^{-3} \mathrm{~s}^{-1}$ \\
\hline$k_{C O 2}$ & $9.72 \times 10^{-2} \mathrm{~s}^{-1}$ \\
\hline$k_{N 2}$ & $7.69 \times 10^{-3} \mathrm{~s}^{-1}$ \\
\hline
\end{tabular}


Table S6.1. Boundary conditions (molar fraction)

\begin{tabular}{|lll|}
\hline$\left[y_{i}^{\text {Steps } 01-03}\right]_{z=0}=y_{\text {feed }, i}$ & $(\mathrm{~S} 1) ; \quad\left[\frac{\partial y_{i}^{\text {Steps } 04-12}}{\partial z}\right]_{z=0}=0$ & (S2) \\
\hline$\left[\frac{\partial y_{i}^{\text {Steps } 01-08}}{\partial z}\right]_{z=L}=0$ & $(\mathrm{~S} 3) ;$ & {$\left[y_{i}^{\text {Step } 09}\right]_{z=L}=\left[y_{i, \text { ave }}^{\text {Step } 06}\right]_{z=L}$} \\
{$\left[y_{i}^{\text {Step } 10}\right]_{z=L}=\left[y_{i, \text { ave }}^{\text {Step } 04}\right]_{z=L}$} & (S5); $\left[y_{i}^{\text {Steps } 11-12}\right]_{z=L}=\left[y_{i, \text { ave }}^{\text {Step } 02-13}\right]_{z=L}$
\end{tabular}

Table S6.2. Boundary conditions (pressure)

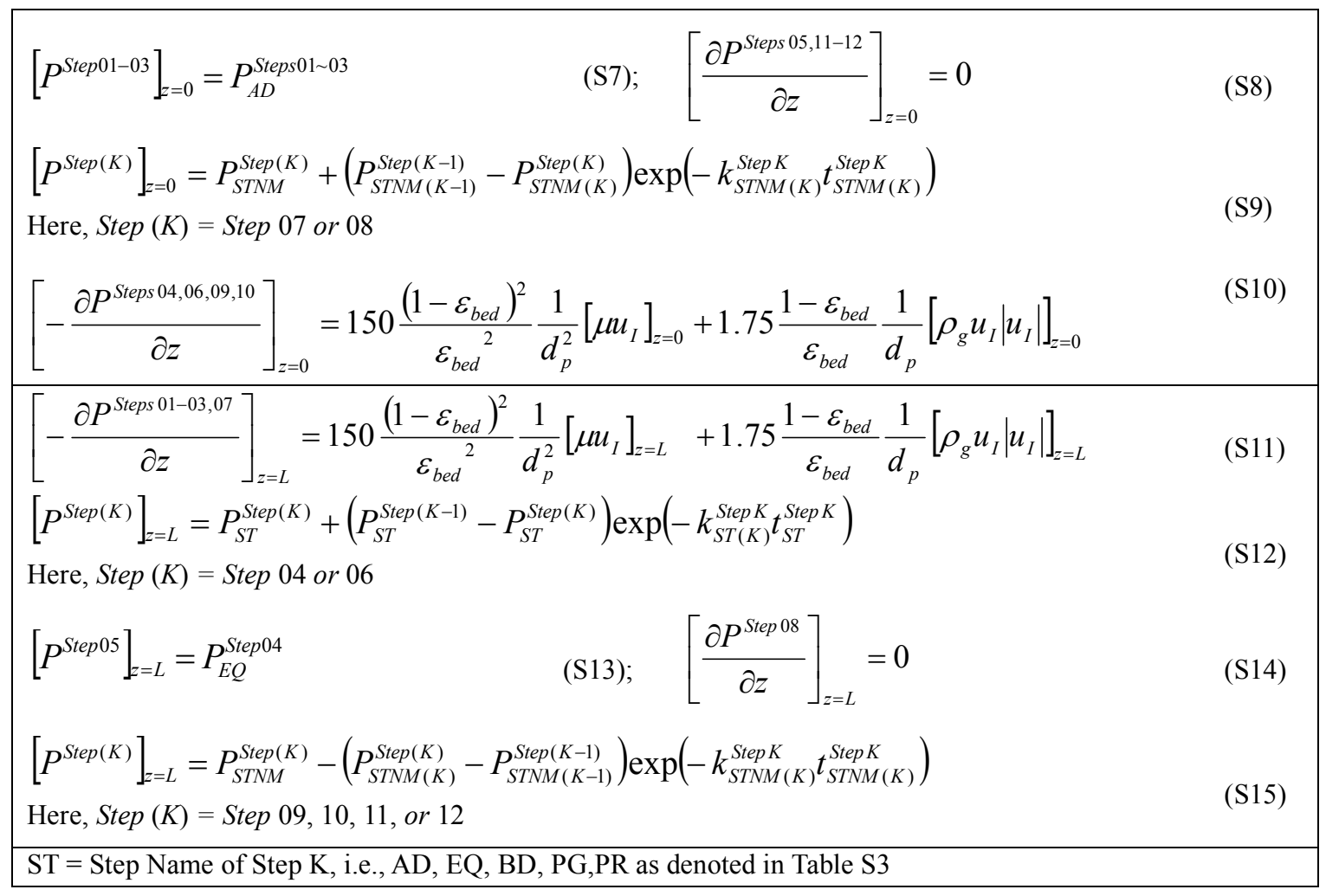


Table S6.3. Boundary conditions (molar flow rate)

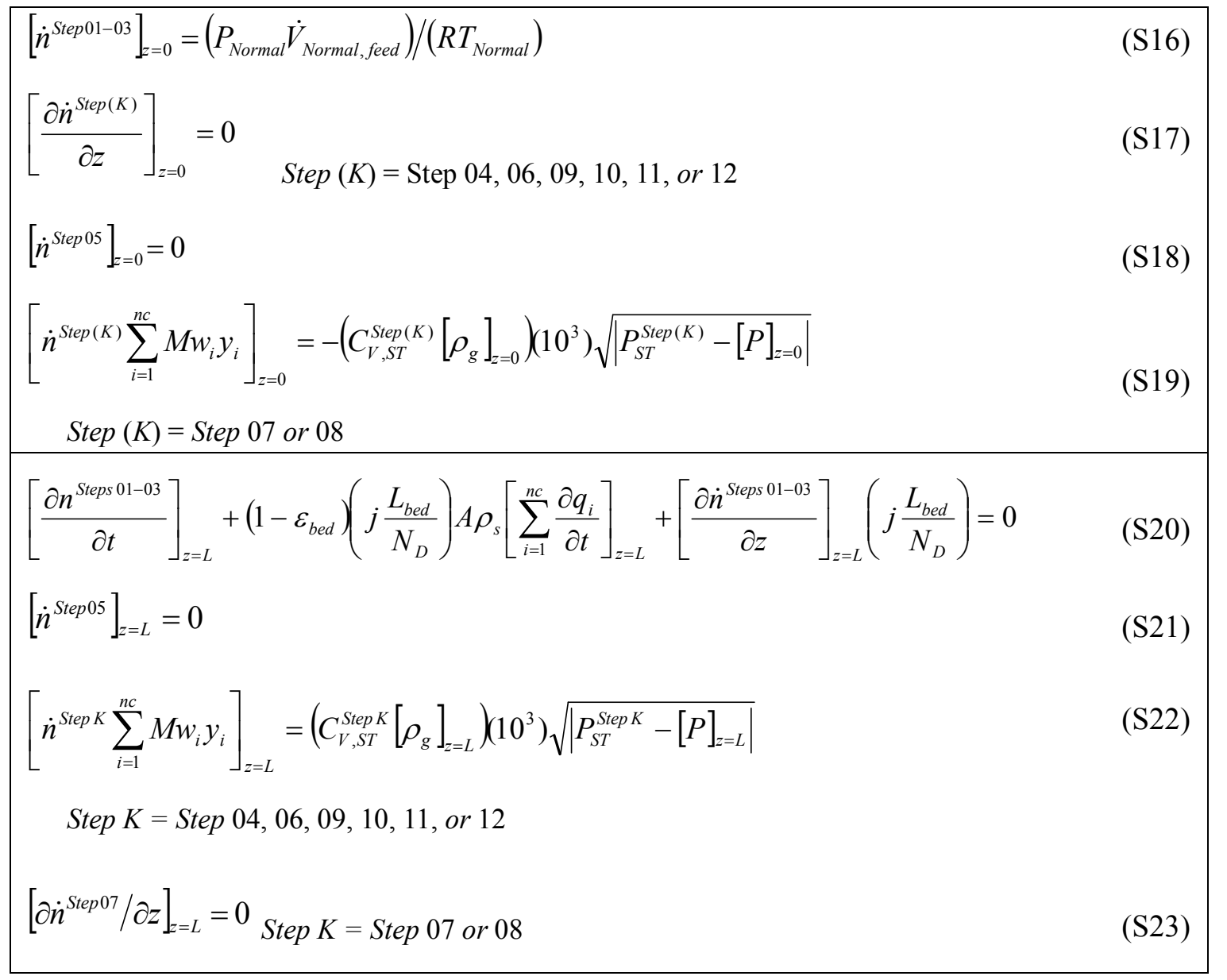

Table S6.4. Boundary conditions (temperature)

\begin{tabular}{|llll|}
\hline$\left[T^{\text {Steps } 01-03}\right]_{z=0}=T_{\text {feed }}$ & $(\mathrm{S} 24) ;$ & {$\left[\frac{\partial T^{\text {Steps } 01-03}}{\partial z}\right]_{z=L}=0$} & (S25) \\
\hline$\left[\frac{\partial T^{\text {Steps 04-12 }}}{\partial z}\right]_{z=0}=0$ & $(\mathrm{~S} 26) ;$ & {$\left[\frac{\partial T^{\text {Steps } 04-12}}{\partial z}\right]_{z=L}=0$}
\end{tabular}


Table S6.5. Boundary conditions (interstitial gas velocity)

\begin{tabular}{|c|c|}
\hline$\left|u_{I}^{\text {Step } 01-03} P\right|_{z=0} A_{\text {void }} T_{\text {Normal }}=[T]_{z=0} \dot{V}_{\text {feed }, \text { Normal }} P_{\text {Normal }}$ & $(\mathrm{S} 28)$ \\
\hline$\left[u_{I}^{\text {Step }(K)}\right]_{z=0}=0 \quad$ Step $(K)=$ Step $04,05,06,09,10,11$, or 12 & (S29) \\
\hline$\left[u_{I}^{\text {Step }(K)}\right]_{z=0} A_{\text {void }}=-\left(C_{V, S T}^{\text {Step }(K)} \sqrt{\left|P_{S T}^{\text {Step }(K)}-[P]_{z=0}\right|}\right)$ & (S30) \\
\hline$\left[u_{I}^{\text {Step } 01-03} P\right]_{z=L} A_{\text {void }}=[\dot{n} T Z]_{z=L} R$ & (S31) \\
\hline$\left[u_{I}^{\operatorname{Step}(K)}\right]_{z=L} A_{\text {void }}=C_{V, S T}^{\operatorname{Step}(K)} \sqrt{\left|P_{S T}^{\operatorname{Step}(K)}-[P]_{z=L}\right|}$ & (S32) \\
\hline$\left[u_{I}^{\text {Step }(K)}\right]_{z=L}=0 \quad$ Step $(K)=$ Step 05,07 , or 08 & (S33) \\
\hline$\left[u_{I}^{\text {Step }(K)}\right]_{z=L} A_{\text {void }}=-C_{V, S T}^{\text {Step } 09} \sqrt{\left|P_{S T}^{\text {Step }(K)}-[P]_{z=L}\right|}$ & (S34) \\
\hline
\end{tabular}


Table S7. Simulation results of conventional approach (II) as to the various values of " $\alpha$ " (laboratory-scale \& pilot-scale)

\begin{tabular}{|c|c|c|c|c|c|c|c|}
\hline Cases & Variable & $\begin{array}{c}\text { Simulation } \\
\text { Conventional } \\
\text { approach (II) } \\
\text { (when } \alpha=1 \text { ) }\end{array}$ & $\begin{array}{c}\text { RME } \\
(\%)\end{array}$ & $\begin{array}{l}\text { Simulation } \\
\text { Conventional } \\
\text { approach (II) } \\
\text { (when } \alpha=0.95 \text { ) }\end{array}$ & $\begin{array}{c}\text { RME } \\
(\%)\end{array}$ & $\begin{array}{l}\text { Simulation } \\
\text { Conventional } \\
\text { approach (II) } \\
\text { (when } \alpha=0.9 \text { ) }\end{array}$ & $\begin{array}{c}\text { RME } \\
(\%)\end{array}$ \\
\hline \multirow{4}{*}{$\begin{array}{c}\text { [Case } 1 \mathrm{~b}] \\
0.68 \mathrm{Nm}^{3} \mathrm{~h}^{-1} \\
\text { (Feed } \\
\text { Flow) }\end{array}$} & Purity $_{\mathrm{CH} 4, \text { ave }}(\%)$ & 96.0851 & 0.078 & 96.1463 & 0.015 & 96.2087 & 0.050 \\
\hline & Recovery $_{C H 4, \text { ave }}(\%)$ & 104.7651 & 10.87 & 99.4771 & 5.704 & 94.1831 & 0.237 \\
\hline & Product Flow $\left(\mathrm{Nm}^{3} \mathrm{~h}^{-1}\right)$ & 0.6608 & 10.65 & 0.6270 & 5.410 & 0.5932 & 0.131 \\
\hline & $t_{\text {sim }}\left(C S S_{\text {cycle }}\right)$ & \multicolumn{2}{|c|}{243 s (10 cycles) } & \multicolumn{2}{|c|}{$170 \mathrm{~s}$ (10 cycles) } & \multicolumn{2}{|c|}{178 s (10 cycles $)$} \\
\hline \multirow{4}{*}{$\begin{array}{c}\text { [Case 2b] } \\
0.56 \mathrm{Nm}^{3} \mathrm{~h}^{-1} \\
\text { (Feed } \\
\text { Flow) }\end{array}$} & Purity $_{C H 4, \text { ave }}(\%)$ & 97.0886 & 0.031 & 97.1296 & 0.011 & 97.1470 & 0.029 \\
\hline & Recovery $_{C H 4, \text { ave }}(\%)$ & 105.0082 & 12.31 & 97.8199 & 5.235 & 94.5904 & 1.879 \\
\hline & Product Flow $\left(\mathrm{Nm}^{3} \mathrm{~h}^{-1}\right)$ & 0.5386 & 12.19 & 0.5015 & 5.058 & 0.4848 & 1.676 \\
\hline & $t_{\text {sim }}\left(C S S_{\text {cycle }}\right)$ & \multicolumn{2}{|c|}{$171 \mathrm{~s}(10$ cycles $)$} & \multicolumn{2}{|c|}{$172 \mathrm{~s}(10$ cycles $)$} & \multicolumn{2}{|c|}{$188 \mathrm{~s}(10$ cycles $)$} \\
\hline \multirow{4}{*}{$\begin{array}{c}\text { [Case 3b] } \\
0.44 \mathrm{Nm}^{3} \mathrm{~h}^{-1} \\
\text { (Feed } \\
\text { Flow) }\end{array}$} & Purity $_{C H 4, a v e}(\%)$ & 97.3076 & 0.18 & 97.3250 & 0.161 & 97.3419 & 0.144 \\
\hline & Recovery $_{C H 4, \text { ave }}(\%)$ & 104.8597 & 13.95 & 99.4562 & 8.672 & 94.0501 & 3.088 \\
\hline & Product Flow $\left(\mathrm{Nm}^{3} \mathrm{~h}^{-1}\right)$ & 0.4249 & 13.92 & 0.4028 & 8.614 & 0.3808 & 2.998 \\
\hline & $t_{\text {sim }}\left(C S S_{\text {cycle }}\right)$ & \multicolumn{2}{|c|}{$188 \mathrm{~s}$ (11 cycles) } & \multicolumn{2}{|c|}{$192 \mathrm{~s}$ (11cycles) } & \multicolumn{2}{|c|}{$181 \mathrm{~s}(11$ cycles $)$} \\
\hline \multirow{4}{*}{$\begin{array}{c}{[\text { Case } 4 \mathrm{~b}]} \\
100 \mathrm{Nm}^{3} \mathrm{~h}^{-1} \\
\text { (Feed } \\
\text { Flow) }\end{array}$} & Purity $_{C H 4, \text { ave }}(\%)$ & 97.1916 & 0.197 & 97.1999 & 0.206 & 97.2072 & 0.213 \\
\hline & Recovery $_{C H 4, \text { ave }}(\%)$ & 105.2489 & 13.43 & 98.4436 & 6.767 & 94.4377 & 2.615 \\
\hline & Product Flow $\left(\mathrm{Nm}^{3} \mathrm{~h}^{-1}\right)$ & 96.8690 & 13.18 & 91.8767 & 7.903 & 86.8840 & 2.319 \\
\hline & $t_{\text {sim }}\left(C S S_{\text {cycle }}\right)$ & \multicolumn{2}{|c|}{703 s (34 cycles) } & \multicolumn{2}{|c|}{658 s (34 cycles) } & \multicolumn{2}{|c|}{757 s (35 cycles) } \\
\hline
\end{tabular}

